Relations industrielles

Industrial Relations

\title{
Scientific Management, Job Redesign and Work Performance, by John E. Kelly, London, Academic Press, 1982, 257 pp., ISBN 012404020-9.
}

\section{Alain Vinet}

Volume 38, numéro 2, 1983

URI : https://id.erudit.org/iderudit/029371ar

DOI : https://doi.org/10.7202/029371ar

Aller au sommaire du numéro

\section{Éditeur(s)}

Département des relations industrielles de l'Université Laval

\section{ISSN}

0034-379X (imprimé)

1703-8138 (numérique)

Découvrir la revue

Citer ce compte rendu

Vinet, A. (1983). Compte rendu de [Scientific Management, Job Redesign and Work Performance, by John E. Kelly, London, Academic Press, 1982, 257 pp., ISBN 012404020-9.] Relations industrielles / Industrial Relations, 38(2), 453-455. https://doi.org/10.7202/029371ar

Tous droits réservés @ Département des relations industrielles de l'Université Laval, 1983
Ce document est protégé par la loi sur le droit d'auteur. L'utilisation des services d’Érudit (y compris la reproduction) est assujettie à sa politique d'utilisation que vous pouvez consulter en ligne.

https://apropos.erudit.org/fr/usagers/politique-dutilisation/ 
améliorer la qualité de la vie au travail s'insère dans l'éventail des interventions propres à réduire le stress à la fois individuel et organisationnel. C'est là une vision fort intéressante des phénomènes mais au plus «réduction mixte» puisque nous sommes loin d'être sûr s'il y a ou non identité de problématique.

Dans un cinquième chapitre, Louise $\mathrm{H}$. Côté-Desbiolles présente un bon résumé d'une étude déjà parue sur les attitudes des travailleurs québécois à l'égard de leur emploi. Au plan de la satisfaction globale, on retrouve comme dans les conclusions de d'autres études similaires, des taux élevés de satisfaction entre $85 \%$ et $90 \%$. Cependant, comme l'indique l'auteur, il faut plutôt s'arrêter aux différences entre les diverses catégories de personnel et les secteurs pour avoir un profil réel de la réalité.

Les chapitres 6 et 7 traitent des liens entre la technologie utilisée dans un processus de fabrication et l'organisation du travail. Claude Duguay reprend alors l'étude des liens entre la qualité de la vie au travail et la productivité pour démontrer que l'impact de la productivité sur la Q.V.T. est avant tout «subordonnée à la qualité de l'appareil de production». Maurice Boisvert et Maurice Lemelin présentent l'approche socio-technique qui tient compte à la fois des systèmes technologiques et des systèmes humains et qui vise l'optimisation conjointe des objectifs des deux systèmes. Les auteurs décrivent également les étapes d'une intervention du Q.V.T. et les quelques instruments de base utilisés. L'un des auteurs du texte, Maurice Boisvert, a déjà publié un volume sur le sujet chez le même éditeur.

La quatrième partie de l'ouvrage (chapitre 8 et 9) traite du lien productité et Q.V.T. dans un contexte de relations du travail, plus précisément, de négociations collectives. Maurice Lemelin fait ressortir les limites de la négociation collective dans la poursuite de l'accroissement de la productivité et de la satisfaction au travail. S'inscrivant dans des rapports de participation conflictuelle, la négociation collective est vécue par les em- ployeurs comme une limite de leurs droits de gérance ou de leur marge de manoeuvre dans leur tentative d'accroître la productivité; elle est vécue par les syndicats comme un moyen prévilégié de contrôle sur le fardeau de travail et sur ses conditions d'exécution. Par ailleurs, la négociation collective demeurant fortement axée sur la réglementation de conditions extrinsèques au travail ne favorisent pas plus un accroissement de la motivation individuelle chez les travailleurs. L'auteur vient tempérer cette vision contraignante de la réalité en citant des exemples montrant une certaine compatibilité entre la négociation collective et l'accroissement de la productivité ou de la satisfaction au travail. Jean-Paul Hétu, président de la C.S.D., retrace la position des syndicats québécois face aux velléités patronales d'accroissement de la productivité dans une perspective Tayloriste et propose une nouvelle approche en s'appuyant sur les principes du courant socio-technique.

Enfin, la dernière partie, qui sert de conclusion à l'ouvrage, reprend le thème des liens entre la productivité et la Q.V.T. pour mettre en exergue les convergences et les divergences entre les deux concepts.

Il est toujours difficile dans un recueil de textes choisis de conserver une certaine continuité à la réflexion sur un thème et le présent ouvrage n'échappe pas à cette difficulté, d'autant plus que les auteurs abordent un sujet qui porte à la controverse. On y retrouve tout de même des éléments de réflexion, des prises de position qui feront dans un avenir immédiat l'objet d'études approfondies.

Laurent BÉLANGER

Université Laval

Scientific Management, Job Redesign and Work Performance, by John E. Kelly, London, Academic Press, 1982, 257 pp., ISBN 012404020-9

Encore un livre sur la restructuration des tâches mais quel livre! Cet ouvrage se distin- 
gue à divers titres: contrairement à la tradition qui s'établit dans ce domaine, il ne s'agit pas d'un assemblage d'articles hétéroclites rédigés par des auteurs différents. John Kelly est l'unique auteur de ce livre et le lecteur est assuré dès les premières pages de l'existence d'une cohérence et d'une continuité entre les chapitres. De plus, Kelly exprime dès le départ le souci d'évaluer de manière critique l'encadrement thérique qui préside au mouvement de restructuration du travail. Et de fait, il dégage la théorie sous-jacente aux expériences diverses de "job redesign", la critique et propose une théorie qui rend mieux compte de l'ensemble du phénomène. Ce n'est pas une mince tâche. Enfin, cet ouvrage repose sur une analyse minutieuse d'une documentation considérable: des dizaines d'expériences sont citées, les points de vue contradictoires sont discutés et la contribution des auteurs qui l'on précédé est évaluée; la bibliographie présentée à la fin de l'ouvrage compte plus de 500 titres.

Kelly offre tout d'abord une lecture nouvelle de l'oeuvre de Taylor. Il s'insurge contre le fait que le mouvement de restructuration des tâches (enrichissement, approche sociotechnique et groupes autonomes) se présente comme une alternative au taylorisme, une innovation en rupture avec la pensée de Taylor. Cette fausse image provient d'une utilisation abusive de certaines remarques de Taylor et d'une ignorance ou d'une méconnaissance de ses autres écrits. À l'aide de nombreuses citations, Kelly corrige le portrait que cette littérature offre de Taylor et démontre que la plupart des idées appliquées aujourd'hui dans la restructuration des tâches étaient présentés dans l'oeuvre de Taylor. De l'OST à la QVT, il y aurait continuité et non rupture. On s'en doutait peut-être mais la démonstration de Kelly est à la fois originale et percutante.

Dès lors, la brèche est ouverte et il devient tentant de considérer comme une idéologie les théories sous-jacentes à la restructuration des tâches. Sans expliciter sa pensée dans des termes aussi radicaux, l'auteur démontre que les théories d'Herzberg et des chercheurs du Tavistock Institute ne suffisent pas à rendre compte des motifs qui prévalent aux expériences de restructuration des tâches et des résultats obtenus.

Conformément à ces théories, des problèmes personnels tels l'absentéisme, le roulement et l'insatisfaction motiveraient les employeurs à restructurer les tâches. Or, l'analyse de plusieurs dizaines de cas indique selon l'auteur que les fluctuations de la demande (pour le produit fabriqué par l'entreprise en cause) et la concurrence entre entreprises rivales sont des facteurs beaucoup plus importants. En d'autres termes, les expériences de restructuration des tâches sont tentées plus fréquemment dans des entreprises qui doivent adapter leurs produits aux exigences variables d'une clientèle diversifiée et dans des secteurs hautement concurrentiels où toute réduction des coûts (et donc des coûts de main-d'oeuvre) est un objectif vital. Kelly démontre ainsi que les motifs de ces expériences sont moins liés à la satisfaction des travailleurs que ne le prétend la théorie et qu'ils correspondent par ailleurs aux préceptes de rationalisation du travail dégagés par Taylor.

Par ailleurs, les théories classiques établissent un lien entre la satisfaction des travailleurs et la productivité de l'entreprise. Aussi, les expériences de restructuration des tâches sont-elles évaluées à l'aide de mesures de productivité et d'indicateurs de satisfaction. En reprenant l'examen de nombreux cas et en se basant sur les travaux d'autres chercheurs, Kelly confirme que ce lien n'a pu être établi empiriquement. Il n'est pas le premier à infirmer cet aspect des théories classiques mais il ajoute aux évaluations précédentes des éléments originaux: son analyse indique que la productivité accrue obtenue dans ces expériences serait principalement expliquée par des incitations monétaires, par des économies (élimination d'emplois) et par un contrôle accru du travail (principe de l'imputabilité). Ces caractéristiques sont encore une fois voisines des méthodes tayloriennes d'amélioration du rendement.

Enfin, contrairement aux théories classiques qui postulent que patrons et employés 
ont un intérêt commun et égal à entreprendre ces expériences, Kelly démontre que les coûts de la restructuration des tâches sont plus élevés pour les employés: emplois perdus, «intensification» du travail, responsabilité accrue, imputabilité, tandis que les avantages qu'ils en retirent censément sont d'ordre psychologique (les avantages des employeurs sont d'ordre économique).

On comprend mieux dans ces conditions les réticences des syndicats à l'égard des diverses formes de restructuration des tâches. L'analyse minutieuse de Kelly met en lumière les principes de rationalisation du travail qui guident ces expériences au-delà du vocabulaire progressiste qui les recouvre. D'ailleurs, l'auteur suggère de dépouiller ce vocabulaire et de remplacer à l'avenir l'expression «groupes autonomes» par "groupes flexibles» et l'expression "enrichissement des tâches» par «intégration verticale des rôles». Quant à l'avenir proprement dit de la restructuration des tâches, Kelly refuse d'adhérer à l'idée voulant qu'il s'agisse d'un phénomène transitoire, bientôt à son déclin. Les employeurs auront toujours intérêt à rationaliser le travail et à diminuer les coûts pour répondre aux impératifs du marché et la concurrence. Des formes de réorganisation du travail qui contribueront à augmenter la productivité subsisteront et se développeront sans cesse.

Alain VINET

Université Laval

Managers and Management in West Germany, by Peter Lawrence, London, Croom Helm, 1980, 202 pp., ISBN 0-85664-912-0

$\mathrm{Au}$ moment où bon nombre d'entreprises américaines connaissent des difficultés importantes, il est de bon ton d'examiner les pratiques de gestion qui ont cours dans des pays où, semble-t-il, les entreprises mondiamieux la crise qui frappe l'économie mondiale. Parmi les pays à la mode, on note le Japon, mais aussi l'Allemagne.
Peter Lawrence est un sociologue anglais qui, dans son ouvrage Managers and management in West Germany nous livre une étude très poussée des managers et de la gestion des entreprises en Allemagne fédérale. Tout en décrivant fort bien l'expérience allemande, l'auteur fait aussi, à l'occasion, des comparaisons avec la situation prévalant dans d'autres pays, notamment en Angleterre et aux États-Unis. Cette approche comparative rend encore plus intéressante la lecture de ce livre qui, par ailleurs, est fort bien construit.

Dans un premier chapitre, l'auteur présente les principales données sur la place de l'Allemagne fédérale parmi les grandes puissances industrielles et dresse un tableau rapide de l'évolution économique de ce pays depuis la fin de la seconde guerre et de sa situation politique et sociale.

Dans les chapitres suivants (deux à huit) l'auteur traite successivement de la structure des entreprises allemandes (forme légale, taille, participation des travailleurs), du «background» des managers allemands (milieu social, qualifications), du caractère du management allemand (aspect collégial, techniques utilisées, etc.), des vues des managers allemands sur l'entreprise et leur "profession" d'administrateur, de la gestion de la production, du contremaître et, enfin, du statut de l'industrie dans la société allemande.

Parmi les observations les plus intéressantes de l'auteur, mentionnons que les managers allemands ne sont pas des diplômés d'écoles de gestion puisqu'il n'y a pas d'institutions de ce type en allemagne. Une grande proportion des managers allemands sont diplômés universitaires et la majorité d'entre eux sont des ingénieurs. La place des ingénieurs dans l'entreprise allemande reflète l'importance qu'on accorde à la production qui a un statut très élevé. Conception du produit, qualité du produit, respect des délais de livraison et service après vente sont les points forts de l'entreprise allemande. L'importance accordée à la «technik» fait que le manager comprend la production. De leur côté, les tra- 